Teng-Yi Huang, $\mathrm{MS}^{2}$ Hsiao-Wen Chung, PhD Ming-Yen Chen, MS $^{3}$ Lung-Hui Giiang, BSC Shy-Chyi Chin, MD Chang-Shin Lee, PhD Cheng-Yu Chen, MD Yi-Jui Liu, PhD ${ }^{4}$

Index terms:

Cerebrospinal fluid, flow dynamics, 167.12144

Cerebrospinal fluid, MR, 167.12144

Magnetic resonance (MR), phase imaging, 163.12144

Published online

10.1148/radiol.2332030884

Radiology 2004; 233:603-608

Abbreviation:

CSF $=$ cerebrospinal fluid

${ }^{1}$ From the Dept of Electrical Engineering National Taiwan Univ, Taipei, Taiwan, ROC (T.Y.H., H.W.C., M.Y.C., Y.J.L.); Dept of Radiology, Tri-Service General Hosp and National Defense Medical Center, 325, Sec 2, ChengKung Road, Neihu 114, Taipei, Taiwan, ROC (T.Y.H., H.W.C., L.H.G., S.C.C., C.S.L., C.Y.C.); and Depts of Biomedical Engineering (M.Y.C.) and Radiological Technology (Y.J.L.), Yuan-Pei Univ of Science and Technology, Hsin-Chu, Taiwan, ROC. Received Jun 5, 2003; revision requested Aug 15; revision received Jan 14, 2004; accepted Feb 17. Supported in part by Tri-Service General Hospital with grant TSCH-C90-52 and by National Science Council with grant NSC902314-B-002-256. Address correspondence to C.Y.C. (e-mail: sandy0928@seed.net.tw).

Authors stated no financial relationship to disclose.

Current addresses:

${ }^{2}$ Dept of Electrical Engineering, National Taiwan Univ of Science and Technology, Taipei, Taiwan, ROC.

${ }^{3}$ Dept of Radiology, Tri-Service General Hosp and National Defense Medical Center, Taipei, Taiwan, ROC.

${ }^{4}$ Dept of Automatic Control Engineering, Feng Chia Univ, Taichung, Taiwan, ROC.

Author contributions:

Guarantor of integrity of entire study, C.Y.C.; study concepts, C.Y.C., H.W.C.; study design, T.Y.H., H.W.C.; literature research, T.Y.H., M.Y.C.; clinical studies, T.Y.H., S.C.C., C.S.L.; experimental studies, T.Y.H., Y.J.L.; data acquisition, T.Y.H., M.Y.C.; data analysis/interpretation, T.Y.H., H.W.C., C.Y.C., L.H.G.; statistical analysis, T.Y.H., H.W.C.; manuscript preparation, T.Y.H., H.W.C.; manuscript definition of intellectual content, T.Y.H., H.W.C., C.Y.C.; manuscript editing, H.W.C., C.Y.C.; manuscript revision/review, H.W.C., C.Y.C., C.S.L.; manuscript final version approval, C.Y.C. ○ RSNA, 2004

\title{
Supratentorial Cerebrospinal Fluid Production Rate in Healthy Adults: Quantification with Two-dimensional Cine Phase-Contrast MR Imaging with High Temporal and Spatial Resolution ${ }^{1}$
}

A method for objectively determining supratentorial cerebrospinal fluid (CSF) production rate is described. The method employs cine phasecontrast magnetic resonance imaging with high temporal and spatial resolution at the level of the aqueduct of Sylvius. Automatic encircling of the aqueduct was accomplished by using a pulsatility-based segmentation approach. Results in 23 healthy adults (18 men, five women; age range, 21-39 years) yielded an average CSF production rate of 305 $\mu \mathrm{L} / \mathrm{min} \pm 145$ (standard deviation); this rate is in good agreement with literature-reported values obtained with invasive ventriculolumbar perfusion measurements. Average operator imprecision was $23.1 \%$ if automatic segmentation was not used. The proposed method is potentially an effective means for measuring supratentorial CSF production rate in humans.

- RSNA, 2004

The production of cerebrospinal fluid (CSF) is known to be associated with an intracranial homeostatic response to changes in the physiologic environment such as changes in blood pressure or heart rate (1). Hence, clinical assessment of the CSF production rate may help in understanding its autoregulatory alterations in response to certain pathophysiologic states, such as aging-related brain atrophy or degeneration that accompanies physiologic environmental changes (2). A typical example of clinical assessment of CSF production rate was shown in a recent study, which revealed that patients with Alzheimer dementia had a reduction in CSF production rate (3). Differences in CSF production may also influence the design of treatment for pediatric patients with hydrocephalus (4). Although existing literature on the human CSF production rate is relatively minimal, the importance of quantitative assessment of this rate should not be overlooked.

Measurements of CSF production rate have been performed by using ventricular catheterization (3), external ventricular drainage (4), or ventriculocisternal perfusion (5) techniques. All of the above approaches, however, are highly invasive. Velocity-sensitive magnetic resonance (MR) imaging involving phasecontrast techniques has recently evolved as an attractive alternative for quantitative assessment of the CSF production rate $(6,7)$, primarily owing to the noninvasive nature of this modality. For instance, a previous investigation involving phase-contrast MR imaging revealed circadian variations in the CSF production rate in healthy adults, with the minimum production rate $(12 \mathrm{~mL} / \mathrm{h} \pm 7$ at about 6:00 PM) being approximately 30\% of the maximum rate $(42 \mathrm{~mL} / \mathrm{h} \pm 2$ at 
2:00 Aм) (8). These results, along with experimental evidence provided by follow-up studies, including one that revealed that administration of the $\beta 1$-receptor antagonist atenolol inhibited CSF production (9), indicate that the phasecontrast MR imaging method seems to have increasing promise in the quantification of CSF production, in spite of being limited to use in obtaining supratentorial measurements.

It is notable, however, that the data reported in the literature for human CSF production rates vary to a great extent, especially for values estimated by using invasive methods (347-370 $\mu \mathrm{L} / \mathrm{min})(2$, $3,10)$ versus values estimated by using MR imaging-based approaches (420-700 $\mu \mathrm{L} / \mathrm{min})(7-9,11-13)$. As a particular example, an aging-related reduction in CSF production that was demonstrated by using the ventricular catheterization method (2) was not revealed in an MR imagingbased study (11), the results of which, on the contrary, showed that there was increased CSF production in aged subjects as compared with production in young adult subjects, suggesting that interindividual factors other than age may play a role.

Although it is possible that the discrepancies may have originated from the very different physical principles of the invasive approach and the MR imaging method, it is also possible that the technical difficulties inherent in the MR imaging method were potential sources of inaccuracies. Specifically, the derivation of the supratentorial CSF production rate in the small cerebral aqueduct of Sylvius, which involves subtraction of two relatively large CSF flow rates in the caudal and cranial directions, is believed to be prone to inaccuracy because of error accumulation. Therefore, an optimization procedure for the noninvasive MR imaging measurement of the CSF production rate is certainly highly desirable. Thus, the purpose of our study was to determine the CSF production rate in healthy young adults by using cine phase-contrast MR imaging with high temporal and spatial resolution.

\section{Materials and Methods}

\section{Subjects and Image Acquisition}

Twenty-three healthy young adults (18 men and five women 21-39 years of age) underwent MR velocity mapping examinations that were performed by using a two-dimensional through-plane phasecontrast technique and a 1.5-T MR imaging system (Vision Plus; Siemens, Erlan- gen, Germany). None of these volunteers had a history of neurologic disease. No specific criteria relating to sex were considered for the recruitment of subjects. The five women were not taking birth control pills and were not imaged during their menstrual periods. Hence, possible hormonal effects on CSF production rate, if present at all, were not expected to affect the results substantially. Before the imaging examinations, the heart rate and blood pressure of the subjects were measured to ensure normal cardiovascular physiologic conditions. Possible neurologic abnormalities were ruled out by performing standard T1-weighted spin-echo, T2-weighted turbo spin-echo, and T2weighted fluid-attenuated inversion-recovery image acquisitions before the phasecontrast acquisition; these images were interpreted by at least one experienced radiologist (S.C.C. or C.Y.C., both of whom had $>10$ years of experience in neurologic MR imaging).

The entire study, including the MR imaging sequence used, was approved by the institutional review board of Tri-Service General Hospital and National Defense Medical Center. Written informed consent was also obtained from all subjects. Note that because individual variations in CSF production rate are relatively large (as will become clear in a later section of this report), we did not attempt to perform a power analysis for experiment design based on the sample size requirement after obtaining some preliminary data. Instead, we aimed to focus on the technical developments involved in achieving an objective and precise measurement method.

The 23 subjects were imaged at night, between 10:00 PM and midnight. The time period for MR imaging examinations was prearranged because the CSF production rate in humans was once reported to exhibit circadian variations (8). For the purpose of velocity mapping, a 6-mm section perpendicular to the distal third of the aqueduct of Sylvius was selected by referencing a midsagittal image, as shown in Figure 1. A two-dimensional cine phase-contrast gradient-echo sequence $\left(45 / 12,20^{\circ}\right.$ flip angle) was used to obtain phase images with bipolar velocity encoding gradients set at a maximum encoding velocity of $20 \mathrm{~cm} / \mathrm{sec}$. Sixtyfour cardiac phases were acquired and rearranged with retrospective electrocardiographic gating (14) to form 30 images that represented sequential phases in a cardiac cycle.

A matrix size of $256 \times 256$ was used with a 10-cm field of view to yield

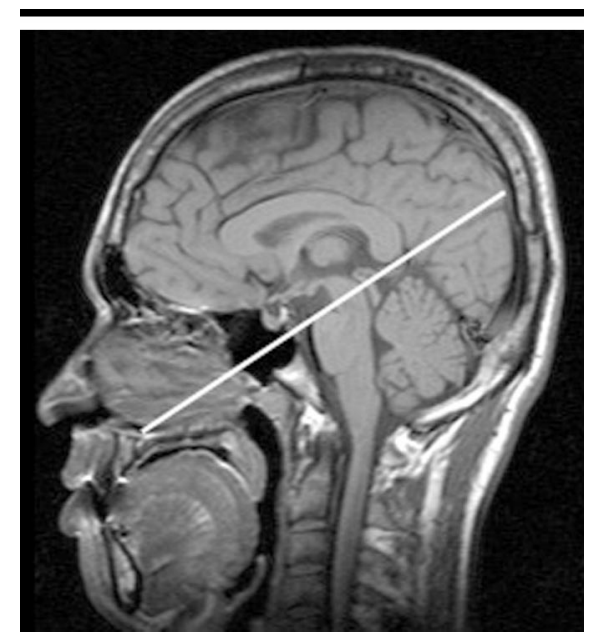

Figure 1. Sagittal T1-weighted spin-echo MR image (repetition time msec/echo time msec, $660 / 20$ ) in healthy 25 -year-old male subject shows location (white line) of section acquired at the distal third of, and perpendicular to, the aqueduct of Sylvius for measurement of CSF production rate.

0.39-mm in-plane spatial resolution. Thus, the cross section of the aqueduct (2-3 $\mathrm{mm}$ in diameter) covered about 16-32 pixels, which was suitable for the analysis method used in this study. A $512 \times 512$ matrix was not chosen owing to our wish to make a compromise between imaging time and signal-to-noise ratio. Aliasing was present but did not affect the subsequent analysis, which was confined to the aqueduct region. The total acquisition time with one signal acquired was about 10 minutes, which was well within the tolerance limits for all of our subjects.

\section{Automatic Aqueduct Segmentation and Image Analysis}

Derivation of CSF production rate was performed with a personal computer (M2NE; ASUS, Taipei, Taiwan) after the phase images were digitally transferred from the console of the MR imaging unit. To minimize the influence of gross brain motion and eddy currents on phase values, the velocity values were first corrected with respect to nearby static tissue (7). A rectangular region of interest of about $1 \times 1 \mathrm{~cm}^{2}$ on the velocity map was then selected (by T.Y.H., who had 6 years of experience in brain MR imaging) to encompass the aqueduct, after which all the analysis steps were executed automatically. Automatic segmentation of the aqueduct was performed by using a method modified from a pulsatilitybased segmentation approach (15). The 
segmentation approach was based on the assumption that the variations in temporal velocity of the aqueductal pixels within a cardiac cycle should strongly correlate with each other. Owing to the nature of the laminar spatial pattern in the absence of turbulent flow, the above assumption was anticipated to be valid.

Note that the absence of turbulent flow in the aqueduct of Sylvius can be shown by calculating the dimensionless Reynolds number $(R)$ with the equation $R=$ $(2 \rho a v) / \eta$, where $\rho$ is the material density of the CSF, $\eta$ is the viscosity of the CSF, $a$ is the radius of the aqueduct, and $v$ is the average flow velocity. Because CSF is a fluid that has density $\left(\rho=1.0 \mathrm{~g} / \mathrm{cm}^{3}\right)$ and viscosity $(\eta=0.01$ poise) similar to those of plasma (16), the maximum Reynolds number of the CSF flow, at a peak velocity of $10 \mathrm{~cm} / \mathrm{sec}$ or lower and in an aqueduct smaller than $3 \mathrm{~mm}$ in radius (note that the parameters used are all worstcase estimations), was around 600. Thus, the estimated maximum Reynolds number was much smaller than the threshold of 2000 for unstable hydrodynamics (17) and strongly suggested an absence of turbulent flow.

With this principle, the temporal velocity profile of the pixel showing the maximum CSF flow velocity was first derived; then, the Pearson correlation coefficients given the above reference velocity profile were calculated for the temporal velocity profiles of all the other pixels. In this manner, a correlation coefficient map, which exhibited better immunity to noise fluctuations than did the original phase-contrast velocity map, was created. The area of the aqueduct was subsequently defined from the correlation coefficient map by using a regiongrowing algorithm (18).

The spatially averaged velocity as a function of cardiac phase was computed by summing all velocity values over the area of the aqueduct and then dividing the summed value by the area for all 30 phase images. The temporal profile of this spatially averaged velocity value was used to investigate the undersampling error, as will be described later. The CSF production rate was calculated as the net CSF volume flowing through the aqueduct in the craniocaudal direction in one cardiac cycle, multiplied by the heart rate. This was achieved by means of numeric integration of the CSF flow velocity throughout the entire cardiac cycle, as well as across the aqueduct area. It will be noticed that because all of the measurements were performed in the aqueduct, the CSF production rate reported in this study reflects supratentorial
CSF secretion from the lateral and third ventricles only.

\section{Operator-dependent Imprecision and Undersampling Error}

To further emphasize the importance of objective segmentation of the small aqueduct, we compared the CSF rate calculated with the method described above with that computed by using a manual definition of the aqueduct on velocity maps. For the latter definition, CSF production rates of the 23 subjects were estimated by using the procedure stated above, with the exception that the aqueduct region was outlined independently by each of three operators (T.Y.H., M.Y.C., and Y.J.L., all of whom had $\geq 5$ years of experience in brain MR imaging). The interoperator agreement was then assessed.

In addition to operator-dependent imprecision, the possible influence of the temporal resolution used at image acquisition on measurement accuracy was also investigated. This latter investigation was performed by using Fourier transformation of the temporal CSF velocity profile, which yielded the power spectrum of CSF velocity at different frequency components (7) (up to the 15th harmonic in this study). A possible error caused by sampling the CSF velocity waveform with insufficient temporal resolution was estimated by examining the magnitude of the high-frequency components relative to the zero frequency component, a process that results in a value that is simply the net CSF flow velocity.

\section{Data and Statistical Analysis}

The interoperator agreement for CSF production rate measurements obtained by using manual aqueduct segmentation was assessed by reporting the range of estimation values in units of percentage of the mean of three measurements (ie, the best estimate when the true value is unknown). The average imprecision was defined as the absolute percentage difference between the individual measurements and the mean value, averaged for all 23 subjects. In addition, Kendall coefficients of concordance were calculated for the three operators to assess the agreement in continuous scoring (19). The Kendall coefficients of concordance ranged from 0.00 to 1.00 , with 1.00 representing excellent agreement among the raters. On the other hand, the mean and standard deviation of the CSF production rates obtained between 10:00 PM and midnight in the 23 healthy young adults were also reported according to data de- rived by using the automatic segmentation algorithm.

\section{Results}

Figure 2 shows the spatial CSF flow profile in a $20 \times 23$-pixel region excerpted from the phase-contrast velocity map in one 25-year-old male subject. The flow patterns for the healthy young adults in this study were largely laminar, thus making it possible to perform automatic outlining of the aqueduct of Sylvius by using the pulsatility-based segmentation algorithm with little difficulty (Fig 3). On the other hand, analysis of the results of calculating the CSF production rate with manual outlining of the aqueduct area revealed that the individual measurements deviated from the mean values by $-59.1 \%$ to $+66.6 \%$.

The average imprecision for all 23 subjects was $23.1 \%$, suggesting that an average uncertainty of $\pm 23.1 \%$ is to be expected in CSF production rate estimation if one is performing manual definition of the small aqueduct on images obtained by using the spatial resolution chosen in our study. The Kendall coefficient of concordance was 0.128 (close to zero), which represents the fact that there was very loose agreement among the three raters. In contrast, with objective automatic segmentation of the aqueduct of Sylvius, operator dependency was obviously absent.

Figure $4 \mathrm{a}$, in which positive velocity represents CSF flow in the craniocaudal direction, shows the temporal profile of the spatially averaged CSF flow velocity in one subject; Figure $4 \mathrm{~b}$ shows the corresponding Fourier coefficients of all of the different frequency components in the form of a power spectrum. The characteristic biphasic (ie, to-and-fro) waveform of the temporal profile $(20,21)$ is clearly discernible in Figure $4 \mathrm{a}$. The biphasic waveform is also appreciable in the power spectrum of Figure $4 \mathrm{~b}$, which shows that the fundamental frequency component (ie, the first harmonic, which corresponds to a heart rate of 60 beats per minute in this case) has a relatively large magnitude compared with the magnitude of the zero-frequency component (ie, the net CSF flow velocity). In fact, for all of the subjects recruited in this study, the sum of the first three harmonics in the power spectra-once described as constituting the essential waveform of the CSF flow profile (7)-was larger than the zero-frequency component (net CSF flow) by a factor of about seven to 11 .

In addition, one will notice that, as 

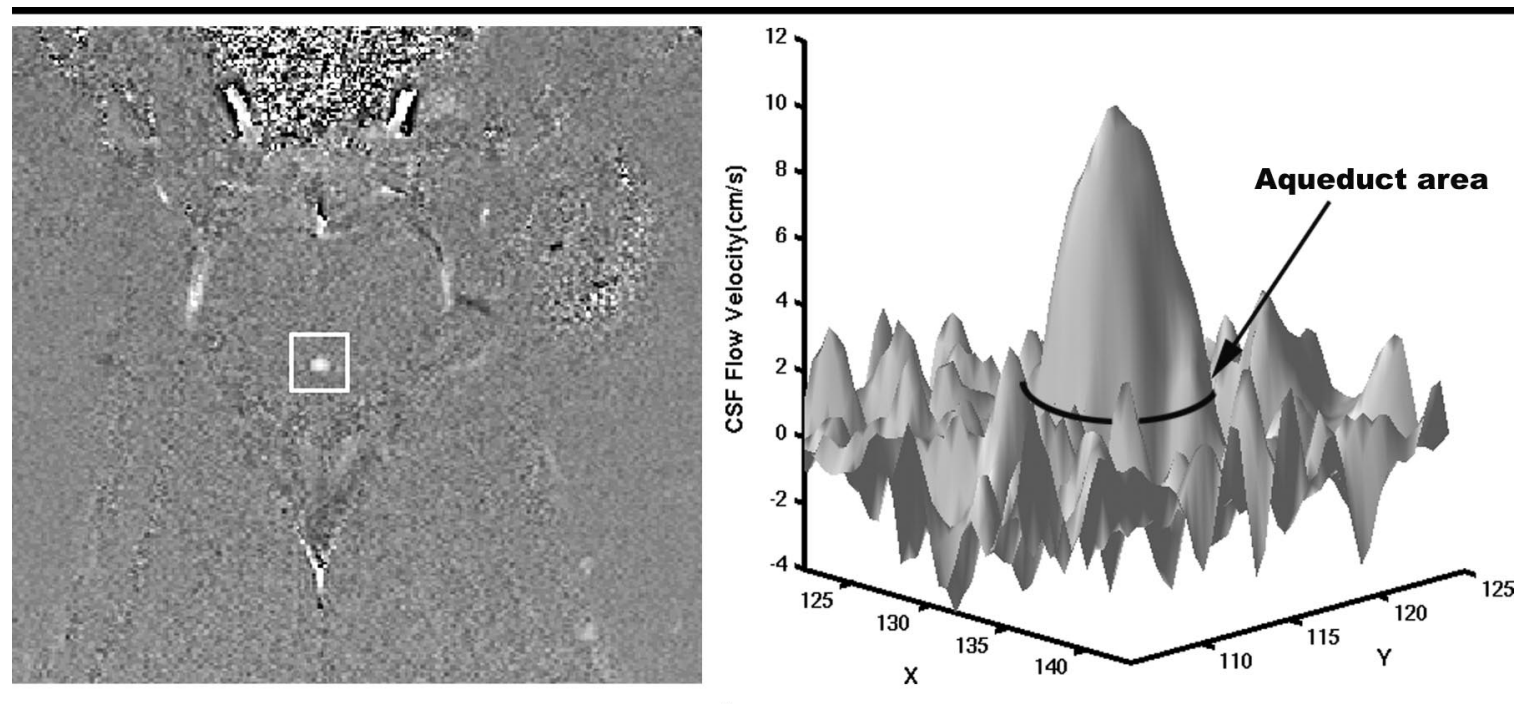

a.

b.

Figure 2. Images illustrate hydrodynamic analysis of CSF in 25-year-old healthy male subject. (a) Phase-contrast gradientecho MR image (45/12, $20^{\circ}$ flip angle) perpendicular to aqueduct of Sylvius shows selection of a rectangular region of interest $(20 \times 23$ pixels in this case) that encompasses the aqueduct; this was the only procedure for hydrodynamic analysis that was performed manually in this study. (b) Graph shows spatial profile of CSF velocity within rectangular region in a; this profile illustrates velocity distribution at one specific cardiac phase. Positive velocity represents CSF flow in the craniocaudal direction.

compared with the zero-frequency component, not all of the higher frequency components in the power spectrum shown in Figure 4b occupied negligible energy. For instance, the seemingly small Fourier coefficient of the 15th harmonic (approximately 0.16) was about onethird of that of the zero-frequency component (approximately 0.49). If the CSF production rate were to be estimated by using phase-contrast MR imaging at a temporal resolution of about eight times the heart rate, the spectral overlapping from undersampling aliasing effects (22) would lead to an error as large as 33\% owing to the 15th harmonic alone.

The mean CSF production rate measured in the 23 subjects by using the automatic analysis algorithm was $305 \mu \mathrm{L} /$ min \pm 145 within the time period from 10:00 PM to midnight. The estimated values were in good agreement with the reported CSF production rates of 347-370 $\mu \mathrm{L} / \mathrm{min}$ obtained by using ventriculolumbar perfusion measurements (10) but were lower than those (420-700 $\mu \mathrm{L}$ / min) obtained by using early MR imaging methods (7-9,11-13).

\section{Discussion}

Despite the potential importance of CSF production rate measurements in the understanding of the response of the intracranial autoregulatory process to environmental changes caused by aging or

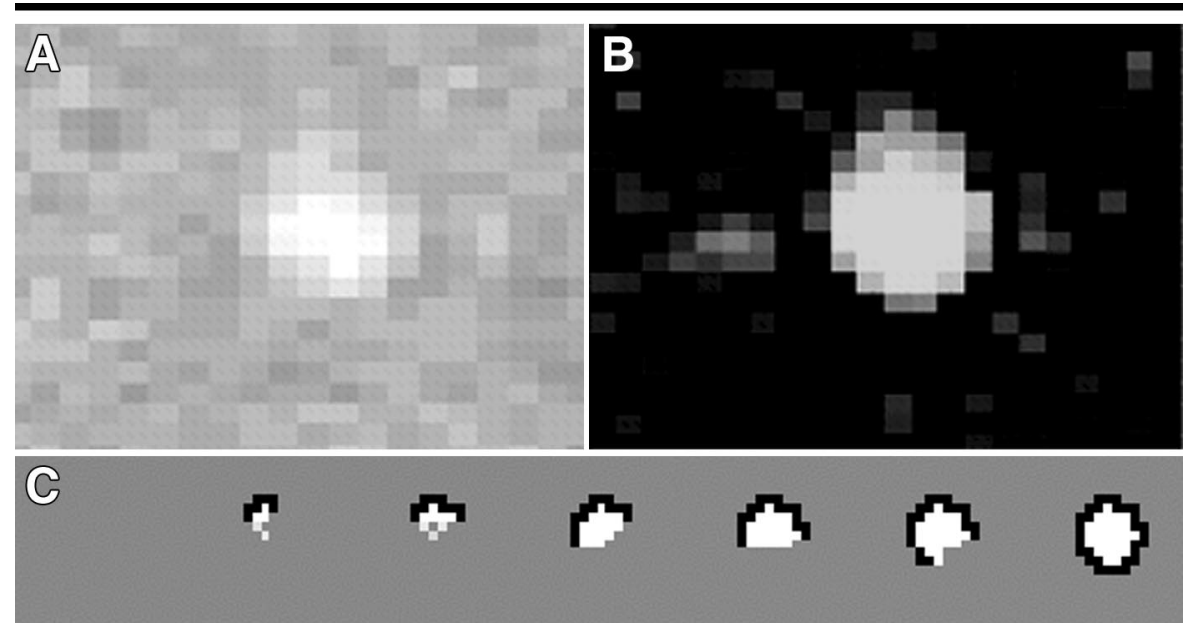

Figure 3. Definition of aqueduct of Sylvius with automatic segmentation involving modified pulsatility-based segmentation algorithm. $A$, Image of region of interest on original phase image (45/12, $20^{\circ}$ flip angle) obtained at maximum velocity in cardiac cycle shows that, although the aqueduct can be identified as the central bright area, a precise and objective definition of the aqueduct is hampered by phase fluctuations outside the aqueductal region. $B$, Image of same region of interest on correlation coefficient map obtained by computing Pearson correlation coefficients of temporal flow patterns on pixel-by-pixel basis. Note that this map is less noisy than the original phase image in $A$ and therefore facilitates objective outlining of the aqueduct. $C$, Series of images show action of region-growing (left to right) algorithm applied to correlation coefficient map in $B$ for automatic segmentation of aqueduct of Sylvius. The aqueduct area defined with this method is subsequently applied to all phase images for hydrodynamic analysis to obtain the CSF production rate.

dementia (1-3), the invasive nature of the procedures conventionally used to calculate such measurements has obviously precluded their wide application in clinical practice. The inherent inaccuracy involved in the extrapolative nature of the ventricular catheterization method, for example, may lead to further uncertainty in the interpretation of the reported values (3). From this aspect, quantitative velocity mapping in the aqueduct of Sylvius performed by using noninva- 


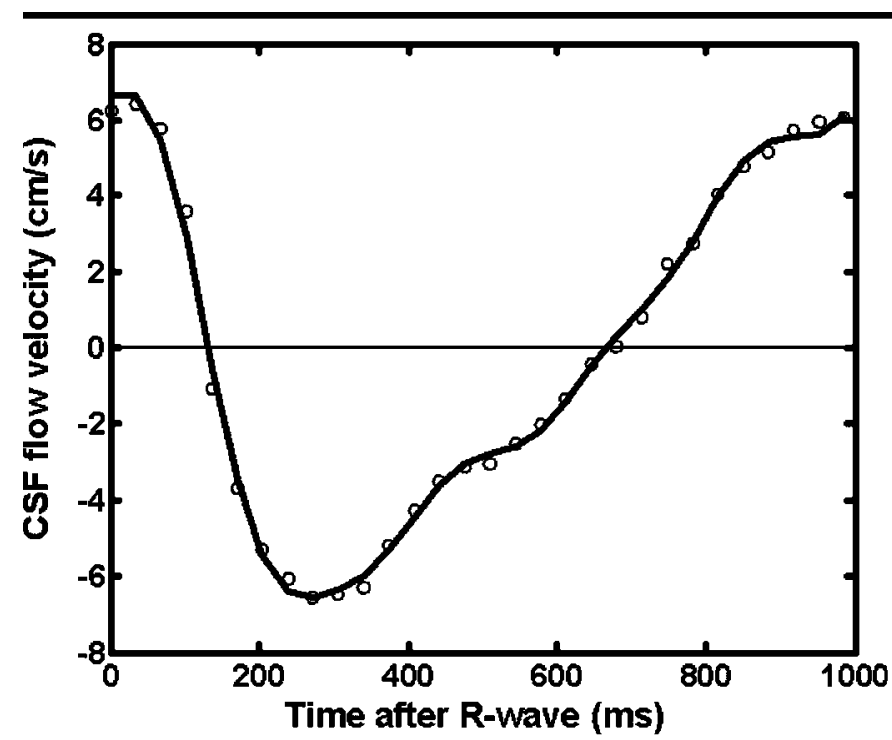

a.

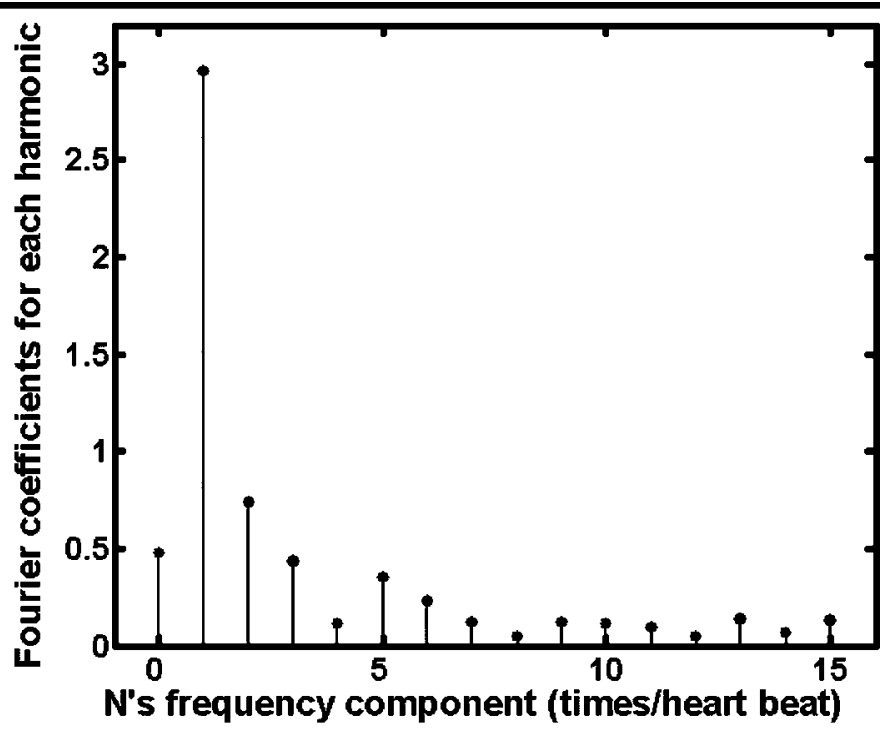

b.

Figure 4. Graphs show temporal pattern of CSF velocity, as well as its frequency spectrum, in 27-year-old healthy male subject. (a) Spatially averaged CSF flow velocity at different cardiac phases. $\bigcirc=$ experimental data, solid line = data after fitting with the first three harmonic components, $R$-wave $=\mathrm{R}$ wave of cardiac cycle. Positive velocity values represent CSF flow in craniocaudal direction. Note that CSF flow is largely biphasic (ie, to and fro), with an average velocity close to zero, suggesting that the derivation of CSF production rate can be prone to inaccuracies because of error accumulation. (b) Fourier coefficients of each harmonic shown as a power spectrum indicate that the zero-frequency component (ie, the net CSF flow) is relatively small compared with the first three harmonics. Also note that owing to the small magnitude of the zero-frequency component, the higher frequency components are not negligible in terms of CSF production rate measurements. $N^{\prime} s$ frequency component $=$ frequency component number.

sive cine phase-contrast MR imaging provides a promising hope $(6-9,11-13)$ that the estimation of CSF production rate can be performed repetitively, without even the need to use ionizing radiation.

It should be understood, however, that measurement of CSF production rate from the aqueduct of Sylvius has some intrinsic difficulties. The CSF in the cerebral aqueduct is characterized by a very small net degree of movement superimposed on a large-amplitude biphasic flow driven by the arterial pulse $(20,21)$. Consequently, most estimation errors, in our opinion, are likely to originate from the accumulation of imprecision (ie, propagation of error) when one is deriving a small quantity from the subtraction of two large numbers. To resolve the discrepancy between MR imaging-derived CSF production rates and literature values obtained by using invasive approaches, it is therefore ultimately important to analyze the imprecision of the technique itself before attempting to attribute the discrepancies as arising from, for example, physiologic variations.

In the present study, variations in measured CSF production rate caused solely by manual definition of the aqueduct area were as large as $23.1 \%$ on average and ranged from $-59.1 \%$ to $+66.6 \%$. Results of previous investigations $(7,14)$ had indi- cated that the calculation of flow rate (or "stroke volume") is less prone to errors resulting from manual selection of regions of interest than is the calculation of average flow velocity, owing to the mutual cancellation of errors in area estimation and errors in average velocity estimation that occurs when one is attempting to derive flow rate. The results of our study, however, suggest that this is not the case for measurements of the "net" CSF production rate in the aqueduct of Sylvius.

Because the net CSF flow through the plane used at MR imaging of the aqueduct of Sylvius is inherently very small, we anticipated that an inclusion of nonmoving background tissue elements in the imaging plane may result in the introduction of relatively large errors through a magnification-of-noise effect. This presence of substantial imprecision in the subjective measurement of CSF production rate has at least two important indications. First, the spatial resolution must be sufficiently high to allow a clear delineation of the cerebral aqueduct, which is only about $2-3 \mathrm{~mm}$ in diameter. Second, dedicated automatic software for reliable and objective aqueduct definition that is immune to image noise is a necessary prerequisite.

If either of the above criteria were not met, measurement inaccuracy could have led to misleading results. It is noted that two of the operators in our study (M.Y.C. and Y.J.L., each of whom had $\geq 5$ years of experience in brain MR imaging) were not informed beforehand of our purpose of interoperator dependency assessment, except for being asked to "try their best" in making reliable measurements. Therefore-additionally given the fact that a high in-plane resolution of $0.39 \mathrm{~mm}$ was used in our study-we regard the large operator variation reported in our study as close to the best-case scenario. On the other hand, because the image analysis algorithm employed in this study was based on pixel-by-pixel examination of the intrinsic similarity of flow patterns, it is therefore operator independent and yields high precision in a reliable manner.

The temporal resolution chosen for MR image acquisition is another important issue influencing accuracy in the measurement of CSF production rate. To understand how temporal resolution influences the accuracy of this measurement, one must realize first that the CSF production rate is determined by the net CSF flow velocity-that is, the zero-frequency component in the temporal velocity profile. The Nyquist sampling theorem states that discrete sampling of a periodic continuous waveform at a frequency of $f_{s}$ essentially results in a repet- 
itive overlay of the spectra spaced $f_{s}$ apart in the frequency domain (22).

In other words, the use of 30 frames within one cardiac cycle in our study allowed the zero-frequency component to be overlapped only by the 30th harmonic of the temporal CSF velocity profile. Figure 4 shows that the magnitudes of the highfrequency components up to the 15th harmonic are at least comparable to the magnitude of the zero-frequency component. Consequently, errors due to undersampling aliasing could result if one uses a temporal resolution lower than about 8 frames within a cardiac cycle. These errors can be prominent, considering once again that the CSF production rate is inherently measured within a very small scale.

The CSF production rates measured in healthy young adults in our study were slightly less than, but in good agreement with, the reported value of $347-370 \mu \mathrm{L} /$ min for ventriculolumbar perfusion measurements (10). This slight discrepancy is within reasonable expectations in that our measurement of the net CSF outflow through the aqueduct of Sylvius only registered CSF secretion in the lateral and third ventricles, while the CSF produced by the choroid plexus in the fourth ventricle may not have been taken into account.

Previous investigators who have evaluated phase-contrast MR techniques have reported values much larger than ours $(8,9,11-13)$. We believe that these previous investigations may have been prone to resolution imprecision and operatorrelated inaccuracy, whereas we achieved high temporal and spatial resolution and used sophisticated image analysis software. Because the percentage of imprecision was not detailed in those reports $(8,9,11-13)$, it is not clear whether those investigators' reported values are significantly larger than our estimations in a statistically meaningful sense. In our study, operator-related imprecision alone ranged from $-59.1 \%$ to $+66.6 \%$, even at MR imaging performed with 30 cardiac phases and $0.39-\mathrm{mm}$ in-plane resolution. Hence, we anticipate that the actual imprecision in investigations involving the use of, for example, six cardiac phases should be even worse. Further studies performed by other independent investigators are needed to resolve the disagreement among MR imaging-based measurements of the CSF production rate.

There were at least two major limitations in our investigation that should be mentioned. First, the CSF production rate measurements were confined to the supratentorial region; hence, the CSF secretion from, for example, the choroid plexus in the fourth ventricle was excluded. Because the relative role of the fourth ventricle, as compared with the role of the lateral and third ventricles, in CSF secretion is largely unknown, our neglect of this portion might have had some influence on the interpretation of the results. We chose to examine supratentorial CSF production simply because the aqueduct of Sylvius, which shows largely biphasic laminar flow, should have served as a convenient target for reliable imaging measurements. Second, the large intersubject variations in CSF production rate among our subjects illustrate the difficulty in the potential usage of CSF production rate as a clinical diagnostic index. This difficulty is understandable, because any disease-related changes in CSF production rate must be large enough to exceed the normal intersubject variations to be statistically meaningful. To resolve this issue, further studies involving patients with CSF-related diseases are certainly needed.

In conclusion, for quantitative studies of the physiologic and pathologic mechanisms of human CSF secretion, it is important to establish a reliable procedure for measuring the CSF production rate. Our results show that two-dimensional cine phase-contrast MR imaging can be used for estimating the CSF production rate-if objective analysis can be performed with images obtained with sufficiently high temporal and spatial resolution. By using our approach, we obtained CSF production rates that are in good agreement with literature values that were obtained by using invasive ventriculolumbar perfusion measurements. Phase-contrast MR imaging is an effective technique that can potentially help resolve unanswered questions relating to human CSF secretion as well as to the relationship between human CSF secretion and intracranial pathophysiologic features.

\section{References}

1. Mendelow AD. Raised intracranial pressure, cerebral edema, hydrocephalus, and intracranial tumors. In: Walton J, ed. Brain's diseases of the nervous system. 10th ed. New York, NY: Oxford, 1993; 144-179.

2. May C, Kaye JA, Atack JR, Schapiro MB, Friedland RP, Rapoport SI. Cerebrospinal fluid production is reduced in healthy aging. Neurology 1990; 40:500-503.

3. Silverberg GD, Heit G, Huhn S, et al. The cerebrospinal fluid production rate is reduced in dementia of the Alzheimer's type. Neurology 2001; 57:1763-1766.

4. Yasuda T, Tomita T, McLone DG, Donovan $\mathrm{M}$. Measurement of cerebrospinal fluid output through external ventricular drainage in one hundred infants and children: correlation with cerebrospinal fluid production. Pediatr Neurosurg 2002; 36:22-28.
5. Javaheri S, Corbett W. Ethanol is a potent inhibitor of canine cerebrospinal fluid production: an acute and reversible effect. Brain Res 1998; 812:91-96.

6. Feinberg DA, Mark AS. Human brain motion and cerebrospinal fluid circulation demonstrated with MR velocity imaging. Radiology 1987; 163:793-799.

7. Thomsen C, Stahlberg F, Stubgaard M, Nordell B. Fourier analysis of cerebrospinal fluid flow velocities: MR imaging study. The Scandinavian Flow Group. Radiology 1990; 177:659-665.

8. Nilsson C, Stahlberg F, Thomsen C, Henriksen O, Herning M, Owman C. Circadian variation in human cerebrospinal fluid production measured by magnetic resonance imaging. Am J Physiol 1992; 262:R20-R24.

9. Nilsson C, Stahlberg F, Gideon P, Thomsen $\mathrm{C}$, Henriksen $\mathrm{O}$. The nocturnal increase in human cerebrospinal fluid production is inhibited by a beta 1-receptor antagonist. Am J Physiol 1994; 267: R1445-R1448.

10. Rubin RC, Ommaya AK, Henderson ES, Bering EA, Rall DP. Cerebrospinal fluid perfusion for central nervous system neoplasms. Neurology 1966; 16:680-692.

11. Gideon P, Thomsen C, Stahlberg F, Henriksen O. Cerebrospinal fluid production and dynamics in normal aging: a MRI phase-mapping study. Acta Neurol Scand 1994; 89:362-366.

12. Gideon P, Stahlberg F, Thomsen C, Gjerris F, Sorensen PS, Henriksen O. Cerebrospinal fluid flow and production in patients with normal pressure hydrocephalus studied by MRI. Neuroradiology 1994; 36:210-215.

13. Gideon P, Sorensen PS, Thomsen C, Stahlberg F, Gjerris F, Henriksen O. Assessment of CSF dynamics and venous flow in the superior sagittal sinus by MRI in idiopathic intracranial hypertension: a preliminary study. Neuroradiology 1994; 36:350-354.

14. Nitz WR, Bradley WG Jr, Watanabe AS, et al. Flow dynamics of cerebrospinal fluid: assessment with phase-contrast velocity MR imaging performed with retrospective cardiac gating. Radiology 1992; 183:395-405.

15. Alperin N, Lee SH. PUBS: pulsatility-based segmentation of lumens conducting nonsteady flow. Magn Reson Med 2003; 49: 934-944.

16. Bloomfield IG, Johnston IH, Bilston LE. Effects of proteins, blood cells and glucose on the viscosity of cerebrospinal fluid. Pediatr Neurosurg 1998; 28:246-251.

17. Jerry BM, William FH. General physics with bioscience essays. 2nd ed. New York, NY: Wiley, 1985; 167-172.

18. Pratt WK. Digital image processing. 3rd ed. New York, NY: Wiley, 2001; 534-547.

19. The R-Project for Statistical Computing. Concordance and reliability package version 1.9.0. Available at: $w w w . r$-project.org/. Accessed August 12, 2004.

20. Bradley WG Jr, Scalzo D, Queralt J, Nitz WN, Atkinson DJ, Wong P. Normal-pressure hydrocephalus: evaluation with cerebrospinal fluid flow measurements at MR imaging. Radiology 1996; 198:523-529.

21. Bradley WG Jr. MR prediction of shunt response in NPH: CSF morphology versus physiology. AJNR Am J Neuroradiol 1998; 19:1285-1286.

22. Oppenheim AV, Willsky AS, Nawab SH. Signals and systems. 2nd ed. Upper Saddle River, NJ: Prentice Hall, 1997; 514-534. 